\title{
The genus Listonella MacDonell and Colwell 1986 is a later heterotypic synonym of the genus Vibrio Pacini 1854 (Approved Lists 1980) - a taxonomic opinion
}

Correspondence
Fabiano L. Thompson
fabiano.thompson@biologia.ufrj.br

tabiano.thompson@iologiaufribr

\author{
Fabiano L. Thompson, ${ }^{1}$ Cristiane C. Thompson, ${ }^{2}$ Graciela M. Dias, ${ }^{1}$ \\ Hiroaki Naka, ${ }^{3}$ Christopher Dubay ${ }^{3}$ and Jorge H. Crosa ${ }^{3}$ \\ ${ }^{1}$ Laboratory of Microbiology, Institute of Biology, Federal University of Rio de Janeiro (UFRJ), Brazil \\ ${ }^{2}$ Laboratory of Molecular Genetics of Microorganisms, Oswaldo Cruz Foundation (FIOCRUZ), Rio \\ de Janeiro, Brazil \\ ${ }^{3}$ Molecular Microbiology \& Immunology, Mail Code L220, Oregon Health \& Science University, \\ 3181 Southwest Sam Jackson Park Road, Portland, OR 97201-3098, USA
}

\begin{abstract}
We analysed the taxonomic position of the genus Listonella based on phylogenetic, genomic and phenotypic data. The species of the genus Listonella were nested within the genus Vibrio according to the $16 \mathrm{~S}$ rRNA gene sequence-based phylogenetic tree. The closest neighbour of Vibrio (Listonella) anguillarum strains LMG $4437^{\top}$ and ATCC 68554 (=strain 775) was Vibrio ordalii LMG $13544^{\top}$, with more than $99.5 \% 16 \mathrm{~S}$ rRNA gene sequence similarity. Furthermore, Vibrio (Listonella) pelagius is highly related to Vibrio splendidus. According to average amino acid identity (AAI), multilocus sequence analysis (MLSA) and Karlin genome signature, the closest neighbour of $L$. anguillarum ATCC 68554 is V. ordalii LMG $13544^{\top}$, with $95 \%$ AAl, $98 \%$ MLSA and 5 in Karlin. V. anguillarum ATCC 68554 and Vibrio cholerae N16961 had 77\% similarity in AAl, $85 \%$ in MLSA and 14 in the Karlin signature. Phenotypic analyses of previously published data for $V$. (L.) anguillarum and $V$. (L.) pelagius revealed that the genus Listonella is extremely similar to the genus Vibrio. $V$. ordalii and $L$. anguillarum strains yielded up to $67 \%$ DNA-DNA hybridization. There are only a few phenotypic features that might be used to discriminate these two species: $L$. anguillarum is positive for the Voges-Proskauer reaction, citrate utilization, starch hydrolysis, lipase activity and acid production from glycerol, sorbitol and trehalose, whereas $V$. ordalii is negative for these traits. We suggest that the genus Listonella is a later heterotypic synonym of the genus Vibrio and propose to use the names Vibrio anguillarum and Vibrio pelagius in place of Listonella anguillarum and Listonella pelagia, respectively.
\end{abstract}

The genus Listonella was created in 1985 using 5S rRNA gene sequences to infer phylogenetic relationships among vibrios and related bacteria (MacDonell \& Colwell, 1985). This study brought useful information to the taxonomy of superfamily I (Vibrionaceae plus Enterobacteriaceae), as it correctly concluded that Vibrio marinus (now Moritella marina in the family Alteromonadaceae), 'Vibrio psychroerythrus' (now Colwellia psychrerythraea in the family Alteromonadaceae) and Aeromonas species (now in the family Aeromonadaceae) were not authentic members of the Vibrionaceae and should be placed into other families. Another significant outcome of this study was the creation

Abbreviations: AAl, average amino acid identity; DDH, DNA-DNA hybridization; MLSA, multilocus sequence analysis.

Two supplementary files are available with the online version of this paper. of the genus Shewanella (now in the family Shewanellaceae; Ivanova et al., 2004).

According to MacDonell \& Colwell (1985), the genus Listonella comprised the species Listonella anguillarum, $L$. damsela and $L$. pelagia. The species $L$. damsela was subsequently reclassified as Photobacterium damselae on the basis of 16S rRNA gene sequence phylogeny (Smith et al., 1991). Although the genus Listonella includes the species $L$. anguillarum and $L$. pelagia, the majority of the work on these micro-organisms uses the species names Vibrio anguillarum and $V$. pelagius, reflecting the actual preference of the field for the Vibrio genus designation. Furthermore, L. anguillarum, L. damsela and L. pelagia were previously classified in the genus Vibrio (Baumann et al., 1971; Bergman, 1909; Love et al., 1981). L. pelagia and V. splendidus show up to $40 \%$ DNA-DNA hybridization (DDH) relatedness 
(Reichelt et al., 1976), whereas the related species Vibrio ordalii and $L$. anguillarum have up to $67 \% \mathrm{DDH}$ relatedness (Schiewe et al., 1981). Because the known error for DDH experiments is around 5\%, these two species are nearly indistinguishable. $L$. anguillarum and $V$. ordalii appeared nested between the core (Harveyi) and Cholerae clades established by Sawabe et al. (2007) using multilocus sequence analysis (MLSA) of nine genes (16S rRNA, rpoA, $r e c A$, fts $Z$, mreB, topA, gyrB, pyrH and gapA), indicating that these species are indeed members of the genus Vibrio. In agreement with the DDH data, L. pelagia appeared within the Splendidus clade (Sawabe et al., 2007).

In this report, we present the results of a taxonomic study based on a larger number of genomic and phenotypic data in order to determine the taxonomic position of $L$. anguillarum and $L$. pelagia. We relied on our recently completed whole-genome sequences of $L$. anguillarum ATCC 68554 (=strain 775) and V. ordalii LMG $13544^{\mathrm{T}}$. Based on this analysis, we propose to use the names Vibrio anguillarum and Vibrio pelagius rather than Listonella anguillarum and Listonella pelagia, respectively.

To determine the exact phylogenetic position of the genus Listonella within the family Vibrionaceae, we performed a phylogenetic reconstruction using the complete $16 \mathrm{~S}$ rRNA gene sequences of several members of this family, as described previously (Thompson et al., 2009). The species of the genus Listonella are nested within the genus Vibrio according to the 16S rRNA gene and MLSA-based phylogenetic trees (Figs 1 and 2; sequence alignments included as Supplementary Material). The closest neighbour of L. anguillarum strains ATCC 68554 and LMG $4437^{\mathrm{T}}$ is $V$. ordalii LMG $13544^{\mathrm{T}}$, with more than $99.5 \%$ $16 \mathrm{~S}$ rRNA gene sequence similarity. The $16 \mathrm{~S}$ rRNA gene sequences obtained from the database (http://www. taxvibrio.lncc.br) and from the whole-genome sequences were indistinguishable. Furthermore, L. pelagia is highly related to V. splendidus. This analysis indicates clearly that the genus Listonella is not distinguishable from the genus Vibrio by means of $16 \mathrm{~S}$ rRNA gene sequence analysis. In general, species of the same genus have $>95 \% 16 \mathrm{~S}$ rRNA gene sequence similarity and form monophyletic groups (Tindall et al., 2010). The definition of a genus requires a polyphasic approach based not only on 16S rRNA gene sequence similarity, but also on several phenotypic and genomic parameters (Tindall et al., 2010).

In order to evaluate the taxonomic position of $L$. anguillarum in more detail, we next determined the genomic similarity of $L$. anguillarum ATCC 68554 and $V$. ordalii LMG $13544^{\mathrm{T}}$ with several members of the family Vibrionaceae, as described previously (Thompson et al., 2009). According to average amino acid identity (AAI), MLSA and Karlin genome signature, the closest neighbour of L. anguillarum ATCC 68554 is V. ordalii LMG 13544 ${ }^{\mathrm{T}}$, with $95 \%$ AAI, $98 \%$ MLSA and 5 in Karlin signature (Table 1). L. anguillarum ATCC 68554 and Vibrio cholerae N16961 had $77 \%$ similarity in AAI, 85\% in MLSA and 14 in the Karlin signature. In addition, the codon usage bias

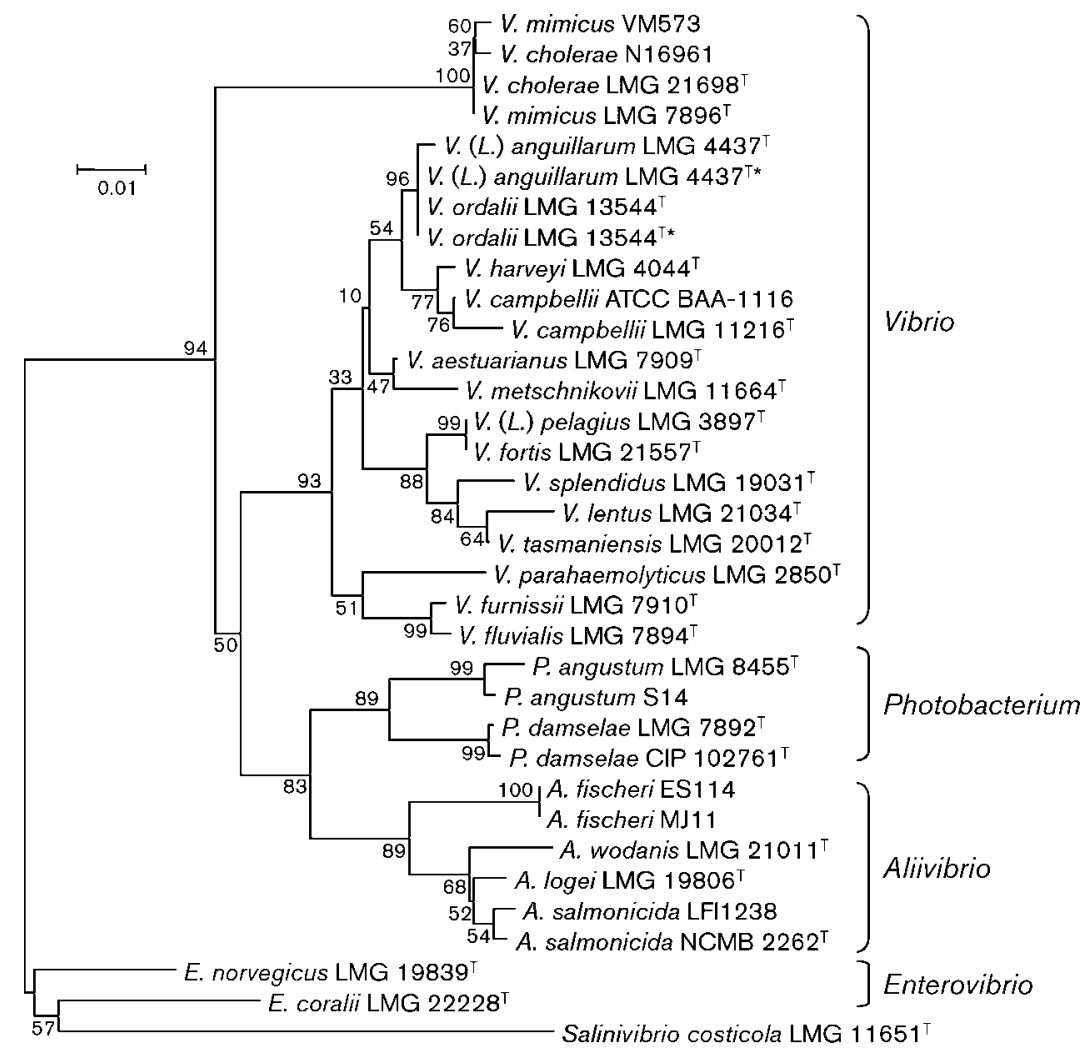

Fig. 1. Phylogenetic tree based on complete $16 \mathrm{~S}$ rRNA gene sequences of several members of the family Vibrionaceae. The tree was reconstructed using the neighbour-joining method and Kimura's two-parameter model. Bar, 1\% sequence difference. Asterisks next to sequences of $L$. anguillarum ATCC 68554 and $V$. ordalii LMG $13544^{\top}$ indicate 16S rRNA gene sequences obtained from whole genome sequences. The alignment used to reconstruct the tree is available as a supplementary file in IJSEM Online. 


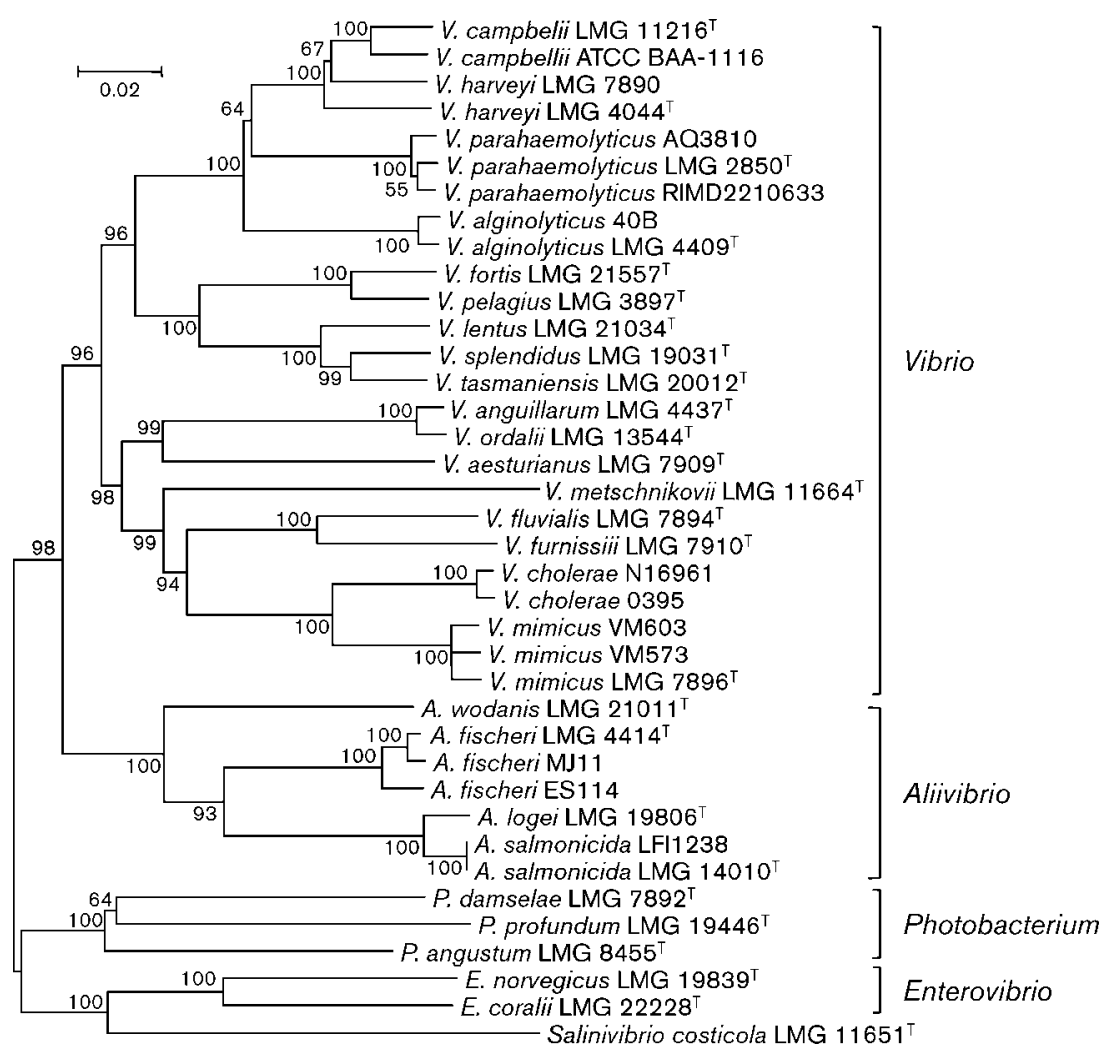

Fig. 2. Phylogenetic tree based on sequences of top $A, \operatorname{rec} A, p y r H, g y r B, m r e B, r p o A$ and fts $Z$ genes of several members of the family Vibrionaceae. The tree was constructed using the neighbour-joining method and $p$ distances. Bar, $2 \%$ sequence difference. The alignment used to reconstruct the tree is available as a supplementary file in IJSEM Online.
(Nc) was 53 for L. anguillarum ATCC 68554 and V. ordalii LMG $13544^{\mathrm{T}}, 52$ for $V$. cholerae N16961, 48 for Photobacterium angustum S14, 49 for Photobacterium damselae CIP $102761^{\mathrm{T}}$ and 51 for Photobacterium profundum SS9. The mean AAI of L. anguillarum towards species of the genera Vibrio and Photobacterium was $78.1 \pm 8.4$ $(n=6)$ and $67 \pm 1.0(n=3)$, respectively, indicating that $L$. anguillarum is indeed closer to the former genus. The mean MLSA similarity of L. anguillarum towards species of the genera Vibrio and Photobacterium was $86.5( \pm 5.8)$ and $79.6( \pm 1.5)$, respectively. These significantly higher values towards the genus Vibrio and the phylogenetic allocation of L. anguillarum within the genus Vibrio reinforce the notion that L. anguillarum is not to be considered a member of a separate genus. New genera of vibrios have been described based on a polyphasic approach, including phylogenetic inferences. For instance, the new genus Alivibrio was created based on the argument that several species (Alivibrio logei, A. salmonicida and A. fischeri), previously classified in the genus Vibrio, may form a separate phylogenetic branch on the outskirts of the genus Vibrio (Urbanczyk et al., 2007). In addition, the codon usage in Alivibrio appeared to be different from that in the genus Vibrio (Thompson et al., 2009). Similar arguments do not apply to L. anguillarum, because the genus Listonella cannot be differentiated from the genus Vibrio in any genomic parameter. The genomic features of Listonella and Vibrio are indistinguishable. In a previous study, we proposed that a Vibrio species is defined as a group of strains that share $>96 \%$ AAI, $>95 \%$ DNA identity in
MLSA and $<10$ in Karlin genome signature (Thompson et al., 2009). Based on this definition, L. anguillarum and $V$. ordalii are highly related species. Strains of the same species and species of the same genus will form monophyletic groups on the basis of MLSA. The different genera of the Vibrionaceae have less than $70 \%$ AAI. These thresholds were proposed based on a limited number of genome sequences, and we highlighted that the analysis of newly sequenced genomes would refine these boundaries for species definition. It would be interesting to determine the taxonomic resolution of AAI, MLSA and Karlin in subsequent studies examining more representative strains of the two species.

Analysis of previously published phenotypic data for $L$. anguillarum and L. pelagia revealed that the genus Listonella is extremely similar to the genus Vibrio. There are only a few phenotypic features that might be used to discriminate $L$. anguillarum and V. ordalii. L. anguillarum is positive for the Voges-Proskauer reaction, citrate utilization, starch hydrolysis, lipase activity and acid production from glycerol, sorbitol and trehalose, whereas $V$. ordalii is negative for these characteristics (Schiewe et al., 1981). Indeed, L. anguillarum and $L$. pelagia have phenotypic features typical of the genus Vibrio (Baumann et al., 1984; Thompson \& Swings, 2006). The most abundant fatty acids are $18: 1 \omega 7 c, 16: 1 \omega 7 c$ and/or iso-15:0 2-OH, $16: 0$, iso-16:0, 14:0, iso-14:0 $3-\mathrm{OH}$, $12: 0,12: 02-\mathrm{OH}$ and $12: 03-\mathrm{OH}$, corresponding to $>80 \%$ of all fatty acids in most vibrios, including $L$. anguillarum and L. pelagia (Austin et al., 1995). The species $L$. 


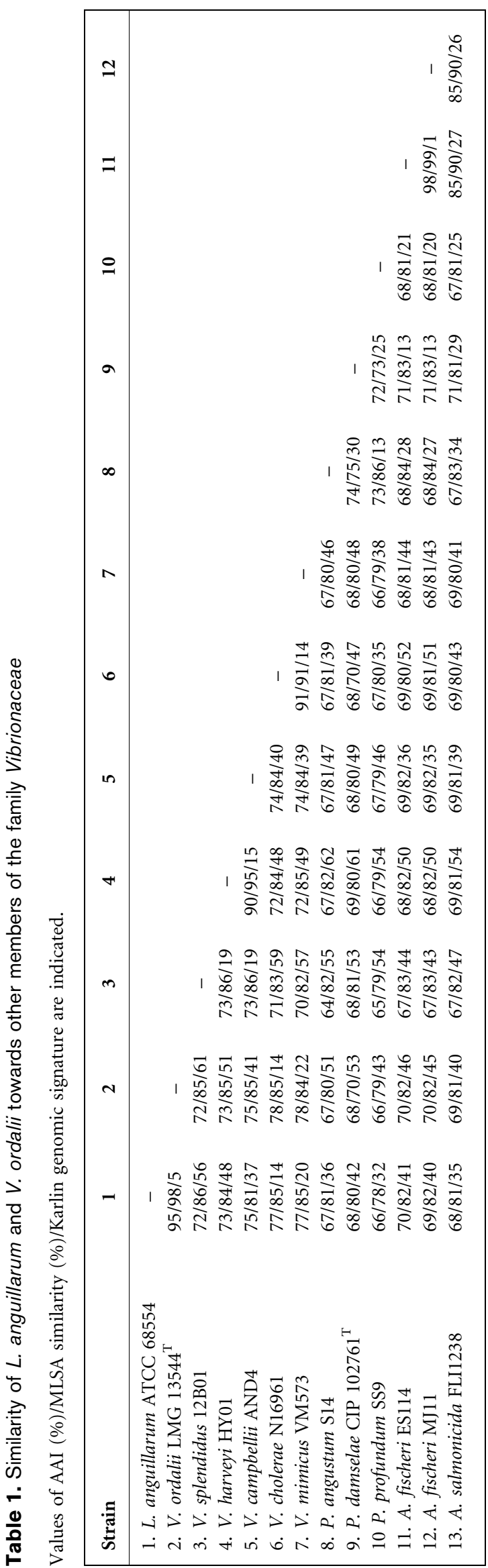

anguillarum is positive for oxidation of dextrin, glycogen, $\mathrm{N}$ acetyl-D-glucosamine, D-fructose, D-glucose and maltose (Austin et al., 1995). Neither phenotypic nor chemotaxonomic data support the separation of the genera Listonella and Vibrio.

The report of the ad hoc committee for the re-evaluation of the species definition in bacteriology (Stackebrandt et al., 2002) suggested that the introduction of innovative approaches in bacterial systematics provides new opportunities for the development and refinement of taxonomy. According to this committee, evolution underpins taxonomy, and the process of doing taxonomy requires adjustments to scientific developments. Our study brings new genomic taxonomic information for the enhancement of the taxonomy of vibrios. Phenotypic, phylogenetic and genomic data presented in this study clearly show that the genus Listonella is indeed nested within the genus Vibrio. Thus, it is clear that these two genera are indistinguishable. Because the genus Vibrio was described first, the genus Listonella is to be considered a later heterotypic synonym of the genus Vibrio. This has been going on long enough, and the time is now right for change in order to avoid further confusion and misleading classifications and, thus, based on our analysis, we propose to use the names Vibrio anguillarum and Vibrio pelagius in place of Listonella anguillarum and Listonella pelagia, respectively.

\section{Acknowledgements}

F.L.T., C.C.T. and G.M.D. thank the CNPq, the US Embassy (Brazil), IFS, CAPES and FAPERJ for support. The contributions of H. N., C. D. and J.H. C. were supported in part by the US Department of Agriculture (\#2007-3560018527) and the National Institutes of Health (RO1-AI19018).

\section{References}

Austin, B., Alsina, M., Austin, D. A., Blanch, A. R., Grimont, P. A. D., Jofre, J., Koblavi, S., Larsen, J. L., Pedersen, K. \& other authors (1995). Identification and typing of Vibrio anguillarum: a comparison of methods. Syst Appl Microbiol 18, 285-302.

Baumann, P., Baumann, L. \& Mandel, M. (1971). Taxonomy of marine bacteria: the genus Beneckea. J Bacteriol 107, 268-294.

Baumann, P., Furniss, A. L. \& Lee, J. V. (1984). Genus I. Vibrio Pacini $1854,411^{\mathrm{AL}}$. In Bergey's Manual of Systematic Bacteriology, vol. 1, pp. 518-538. Edited by N. R. Krieg \& J. G. Holt. Baltimore: Williams \& Wilkins.

Bergman, A. M. (1909). Die rote Beulenkrankheit des Aals. Ber Bayerischen Biol Versuchs-Station 2, 10-54 (in German).

Ivanova, E. P., Flavier, S. \& Christen, R. (2004). Phylogenetic relationships among marine Alteromonas-like proteobacteria: emended description of the family Alteromonadaceae and proposal of Pseudoalteromonadaceae fam. nov., Colwelliaceae fam. nov., Shewanellaceae fam. nov., Moritellaceae fam. nov., Ferrimonadaceae fam. nov., Idiomarinaceae fam. nov. and Psychromonadaceae fam. nov. Int J Syst Evol Microbiol 54, 1773-1788.

Love, M., Teebken-Fisher, D., Hose, J. E., Farmer, J. J., III, Hickman, F. W. \& Fanning, G. R. (1981). Vibrio damsela, a marine bacterium, 
causes skin ulcers on the damselfish Chromis punctipinnis. Science 214, $1139-1140$.

MacDonell, M. T. \& Colwell, R. R. (1985). Phylogeny of the Vibrionaceae, and recommendation of two new genera, Listonella and Shewanella. Syst Appl Microbiol 6, 171-182.

Reichelt, J. L., Baumann, P. \& Baumann, L. (1976). Study of genetic relationships among marine species of the genera Beneckea and Photobacterium by means of in vitro DNA/DNA hybridization. Arch Microbiol 110, 101-120.

Sawabe, T., Kita-Tsukamoto, K. \& Thompson, F. L. (2007). Inferring the evolutionary history of vibrios by means of multilocus sequence analysis. J Bacteriol 189, 7932-7936.

Schiewe, M. H., Trust, T. J. \& Crosa, J. H. (1981). Vibrio ordalii sp. nov.: a causative agent of vibriosis in fish. Curr Microbiol 6, 343-348.

Smith, S. K., Sutton, D. C., Fuerst, J. A. \& Reichelt, J. L. (1991). Evaluation of the genus Listonella and reassignment of Listonella damsela (Love et al.) MacDonell and Colwell to the genus Photobacterium as Photobacterium damsela comb. nov. with an emended description. Int J Syst Bacteriol 41, 529-534.
Stackebrandt, E., Frederiksen, W., Garrity, G. M., Grimont, P. A. D., Kämpfer, P., Maiden, M. C. J., Nesme, X., Rosselló-Mora, R., Swings, J. \& other authors (2002). Report of the ad hoc committee for the reevaluation of the species definition in bacteriology. Int J Syst Evol Microbiol 52, 1043-1047.

Thompson, F. L. \& Swings, J. (2006). Taxonomy of the vibrios. In The Biology of Vibrios, pp. 29-43. Edited by F. L. Thompson, B. Austin \& J. G. Swings. Washington, DC: American Society for Microbiology.

Thompson, C. C., Vicente, A. C., Souza, R. C., Vasconcelos, A. T., Vesth, T., Alves, N., Jr, Ussery, D. W., lida, T. \& Thompson, F. L. (2009). Genomic taxonomy of vibrios. BMC Evol Biol 9, 258.

Tindall, B. J., Rosselló-Móra, R., Busse, H. J., Ludwig, W. \& Kämpfer, P. (2010). Notes on the characterization of prokaryote strains for taxonomic purposes. Int J Syst Evol Microbiol 60, 249-266.

Urbanczyk, H., Ast, J. C., Higgins, M. J., Carson, J. \& Dunlap, P. V. (2007). Reclassification of Vibrio fischeri, Vibrio logei, Vibrio salmonicida and Vibrio wodanis as Alivibrio fischeri gen. nov., comb. nov., Alivibrio logei comb. nov., Aliivibrio salmonicida comb. nov. and Aliivibrio wodanis comb. nov. Int J Syst Evol Microbiol 57, 2823-2829. 